\title{
Early or late parenteral nutrition for the sick preterm infant?
}

\author{
K G Brownlee, E J Kelly, P C Ng, S C Kendall-Smith, P R F Dear
}

\begin{abstract}
No one doubts that good nutrition is an important component of neonatal intensive care, nor that this can only be accomplished by the use of intravenous fat. With regard to the effects of nutrition on bronchopulmonary dysplasia, however, we are facing a dilemma. On the one hand there is the suggestion that inadequate nutrition increases the severity of bronchopulmonary dysplasia and on the other that the use of intravenous fat predisposes to it.

In an attempt to narrow the area of uncertainty we randomly allocated 129 infants of less than $1750 \mathrm{~g}$ birth weight to receive either early or late lipid containing parenteral nutrition.

The median duration of ventilation support in the 'early' group was 8.5 days and in the 'late' group eight days; this was not significantly different.

(Arch Dis Child 1993; 69: 281-283)
\end{abstract}

Very immature infants are born with extremely limited endogenous energy reserves and there is a pressing need to supplement these as soon as possible after birth. It can be calculated that the average $1000 \mathrm{~g}$ infant has enough stored energy to survive for about five days. ${ }^{1}$ The addition of a $10 \%$ dextrose infusion lengthens this theoretical survival time only to about 10 days, indicating that even with this external energy source endogenous reserves would soon be eroded to serve the needs of essential basal metabolism.

In addition to securing survival, modern neonatal medicine has as its aim the maximisation of developmental potential. Recent evidence shows that this too is significantly related to the quality of early nutrition. ${ }^{2}$

There are therefore two good reasons to explore ways of implementing a nutritional regimen which minimises dependence on endogenous energy stores and, as soon as possible, begins to meet the requirements of the nervous system for the necessary substrates for growth.

The provision of complex nutrients to sick preterm infants who are struggling to adapt to extrauterine life carries, in common with almost all medical interventions, the possibility of doing more harm than good and must be carefully evaluated so that decisions about its use are appropriately informed. Published work about the early provision of parenteral nutrition to such infants ${ }^{3-6}$ certainly raises some concerns about safety, particularly of intravenous lipids, but is insufficient to inform clinical decision making. There is currently sufficient professional equipoise about the value of the early introduction of lipids into parenteral nutrition regimens to justify randomised comparative studies of different regimens.

\section{Subjects and methods}

Preterm infants admitted to the Regional Neonatal Intensive Care Unit at St James's University Hospital, Leeds between January 1990 and November 1991 were considered for this trial.

Infants were allocated to one of two groups when they were between 12 and 24 hours of age. Allocation was random but included stratification according to gestation and the severity of lung disease, as determined by the pressure/shunt product at 12 hours of age. The pressure/shunt product is a validated index of the severity of respiratory distress syndrome ${ }^{7}$ which takes into account the inspired oxygen, the mean airway pressure, and the arterial oxygen tension.

\section{SUBJECTS}

Infants were entered into the study if their birth weight was equal to or less than $1750 \mathrm{~g}$, if they were still requiring intermittent positive pressure ventilation (IPPV) at 12 hours of age, and had radiographic features of respiratory distress syndrome. Infants with severe congenital abnormalities or pulmonary hypoplasia were excluded from the study.

A total of 129 infants were recruited into the trial. Those infants randomised into the early group received parenteral nutrition within the first 36 hours; those in the late group received parenteral nutrition on the sixth complete day.

\section{METHODS}

In the two groups parenteral nutrition followed our standard regimen. Intralipid $20 \%$ (KabiVitrum) and either Vamin 9 glucose or Vamin Infant (KabiVitrum) were started at a dose of $0.5 \mathrm{~g} / \mathrm{kg} /$ day and increased daily by this amount to a maximum of $3.5 \mathrm{~g} / \mathrm{kg} /$ day. Lipid infusions were continuous over 24 hours. The fluid regimen was the same for the early and late groups; infants were started on 75 $\mathrm{ml} / \mathrm{kg} /$ day of $10 \%$ dextrose solution and this was increased daily in increments to $165-180$ $\mathrm{ml} / \mathrm{kg} /$ day. 
Table 1 Characteristics of the two groups

$\begin{array}{cc}\begin{array}{l}\text { Early total parenteral } \\ \text { mutrition }(n=63)\end{array} & \begin{array}{l}\text { Late total parenteral } \\ \text { mutrition }(n=66)\end{array} \\ 29(23-33) & 29(24-36) \\ 1144(539-1748) & 1147(415-1647) \\ 478(292) & 518(301)\end{array}$

Median (range) gestation (weeks) Median (range) birth weight (g) Mean (SD) pressure shunt product

$1147(415-1647)$

Table 2 Nutrient intakes of the two groups during the first seven complete days of life
Results

Table 1 gives the characteristics of the two groups of infants. There was no significant difference at the $5 \%$ level of significance between the two groups when compared for gestation, birth weight, and pressure/shunt product at 12 hours of age.

Twenty five $(19 \%)$ infants died after entry into the trial. There were $11(17 \%)$ deaths in the early nutrition group and $14(21 \%)$ deaths in the late nutrition group. This difference was not statistically significant. Seven $(5 \%)$ infants died as the direct result of chronic lung disease (oxygen dependent at 28 days), four in the early and three in the late group. Twelve $(9 \%)$ infants died as a result of severe respiratory distress syndrome or pulmonary interstitial emphysema; four (3\%) infants died after sepsis and one set of twins of congenital abnormalities. These infants were excluded from further analysis.

Table 2 gives the nutritional intake received by the two groups during the first seven complete days of life. Most (116 (90\%)) of the infants received parenteral nutrition only. A few $(13(10 \%))$ were started on enteral feeds within the first seven days and these infants are included in the table only while receiving full parenteral nutrition.

Table 3 compares the two groups with respect to the incidence and severity of chronic lung disease as shown by the duration of assisted ventilation and oxygen treatment. There was no significant difference between the two groups for any of these indices.

Forty $(31 \%)$ infants developed chronic lung disease defined as oxygen dependency at 28 days of age. Twenty of these were in the early parenteral nutrition group and 20 in the late parenteral nutrition group.

Table 3 also compares the two groups for age at discharge, average weight gain per day during the stay in hospital, and average weight gained by 2 weeks of age. There was no significant difference for any of these measures.

Data were also collected on the incidence of patent ductus arteriosus, intraventricular haemorrhage, and clinically important jaundice requiring treatment; there was no significant difference between the groups for these complications.

Table 4 gives the plasma triglyceride concentrations in the two groups or infants. blood gases were sampled at regular intervals determined by the clinical status of the infant.

Infants were weighed at birth, 2 weeks of age, 4 weeks of age, and at discharge using electronic baby scales accurate to within $5 \mathrm{~g}$.

In January 1991 we began to use surfactant regularly as part of the Osiris trial. The use of random allocation ensured that equal numbers of infants in each group received surfactant.

Statistical analysis of results was performed using the Mann-Whitney U test for nonnormally distributed data and Student's $t$ test for normally distributed data.

Approval for the trial was obtained from the local research (ethics) committee and informed consent was obtained from parents before entry to the study.

\section{Discussion}

The components of parenteral nutrition that has given rise to most concern in neonatology are lipids, which are essential if the regimen is to contain sufficient energy to achieve the outlined objectives.

The use of intravenous lipids in preterm infants has been reported to be associated with a short term deterioration in pulmonary function, increased pulmonary vascular tone, and the deposition of lipid particles in alveolar macrophages and capillaries. ${ }^{489}$ All these effects might be expected to increase ventilatory requirements and so increase the incidence and severity of chronic lung disease. 
None of this work helps to determine the influence of the early introduction of parenteral lipids on any measure of medium or long term outcome. Two research studies, however, have suggested potentially serious medium term clinical complications of using intravenous lipids early in the course of neonatal intensive care. ${ }^{34}$

The first, a small randomised trial from Chicago 4 allocated 42 infants to receive intravenous lipids either before or after 5 days of age and showed a significant increase in the incidence and severity of chronic lung disease in those infants given lipids earlier. We struggle to relate the findings of that study to our own practice as the duration of assisted ventilation and oxygen treatment in the Chicago cohort was so much greater than in our own group of infants of similar gestation and weight.

The second study, a large retrospective analysis performed in Liverpool, ${ }^{3}$ showed a statistically significant association between the earlier introduction of intravenous lipids and an increased incidence and severity of chronic lung disease, using the powerful tool of logistic regression. This finding certainly indicates the need for a prospective randomised control trial.

One other small randomised trial of early versus late lipids did not show an adverse effect of early lipids on the development of chronic lung disease. ${ }^{10}$

Our study did not show any detrimental effect on the course of neonatal lung disease of beginning a lipid-containing parenteral nutrition regimen within the first 36 hours of life as opposed to on the sixth day of life. This is despite the fact that the study had a $90 \%$ power to show a three day difference in the duration of IPPV, or a one week difference in the duration of oxygen treatment at the $5 \%$ level of significance. We chose these magnitudes of difference as being clinically significant. A larger trial would be needed to have a reasonable chance of detecting smaller differences.
In addition to looking for detrimental effects, it is also important to define and quantify the potential benefits of early and effective nutrition. Evidence is becoming available about the importance of early nutrition on long term growth and development. Lucas and coworkers have published several papers suggesting that the quality of nutrition over a brief, but perhaps critical, postnatal period has developmental consequences that persist into infancy. ${ }^{2}$

We did not show any significant benefit from early parenteral nutrition in terms of weight gain, though the trend at 2 weeks of age was in favour of the early nutrition group. We were not surprised by this as the difference in the intake of nutrients was relatively small and short lived. This does not, however, preclude the possibility of significant benefit if the extra nutrients were utilised preferentially by the central nervous system, as is usual under conditions of starvation at this stage of development.

1 Heird WC, Driscoll JM, Schullinger JN, Burton G, Winters RW. Intravenous alimentation in pediatric patients. 7 Pediatr 1972; 80: 351-72.

2 Lucas A, Morley R, Cole TJ, et al. Early diet in preterm babies and developmental status in infancy. Arch Dis Child 1989; 64: 1570-8.

3 Cooke RWI. Factors associated with chronic lung disease in preterm infants. Arch Dis Child 1991; 66: 776-9.

4 Hammerman C, Aramburo MJ. Decreased lipid intake reduces morbidity in sick premature neonates. $\mathcal{f}$ Pediatr 1988; 113: $1083-8$.

5 Pereira GR, Fox WW, Stanley CA, Baker L, Schwartz JG. Decreased oxygenation and hyperlipemia during intravenous fat infusions in premature infants. Pediatrics 1980; 66: $26-30$

6 Levene MI, Batisti O, Wigglesworth JS, et al. A prospective study of intrapulmonary fat accumulation in the newborn lung following intralipid infusion. Acta Paediatr Scand 1984; 73: 454-60.

7 Rojas J, Roberts S, Green L, et al. A quantitative model for hyaline membrane disease. Pediatr Res 1982; 16: 35-9.

8 Shulman RJ, Langston C, Schanler RJ. Pulmonary vascular lipid deposition after administration of intravenous fat to infants. Pediatrics 1978; 79: 99-102.

9 Friedman Z, Marks KH, Maisels MJ, Thorson R, Maeye R. Effect of parenteral fat emulsion on the pulmonary and reticuloendothelial systems in the newborn infant. Pediatrics 1978; 61: 694-8.

10 Gilbertson N, Kovar IZ, Cox DJ, Crowe L, Palmer NT. Introduction of intravenous lipid administration on the first day of life in the very low birthweight neonate. f Pediatr 1991; 119: 615-23. 